\title{
GAMIFICACIÓN EN EDUCACIÓN SUPERIOR. REVISIÓN DE EXPERIENCIAS REALIZADAS EN ESPAÑA EN LOS ÚLTIMOS AÑOS
}

\author{
GAMIFICATION IN HIGHER EDUCATION. REVIEW OF EXPERIENCES \\ CARRIED OUT IN SPAIN IN RECENT YEARS
}

\section{GAMIFICAÇÃO NA EDUCAÇÃO SUPERIOR. REVISÃO DE EXPERIÊNCIAS REALIZADAS NA ESPANHA NOS ÚLTIMOS ANOS}

\author{
Santiago Alonso-García \\ Universidad de Granada, España \\ https://orcid.org/0000-0002-9525-709X \\ salonsog@ugr.es
}

José Antonio Martínez-Domingo

Universidad de Granada, España https://orcid.org/0000-0002-4976-7320 josemontejicar@correo.ugr.es

Blanca Berral-Ortiz

Universidad de Granada, España https://orcid.org/0000-0001-8139-8468

Juan Carlos De la Cruz-Campos

Universidad de Granada, España https://orcid.org/0000-0002-9263-6799 juancarlosdelacruz@ugr.es

Resumen: La gamificación está cobrando impulso como innovación educativa para motivar e involucrar a los estudiantes en su aprendizaje. El giro digital en la educación española manifiesta el problema de la motivación, compromiso y disfrute de los estudiantes, pero hasta ahora, no hay investigaciones que revelen el papel de la gamificación en los estudios universitarios realizados en España, ya que esta metodología se ha desarrollado recientemente. Nuestra investigación realiza un análisis de experiencias en educación superior en España en los últimos años. Parte de un diseño de mapeo sistemático de artículos en 'Web of Science', con los siguientes criterios de inclusión: estudios empíricos de gamificación establecidos en España, publicados entre 2016 y 2020 y realizados en entornos reales. La búsqueda inicial arrojó 332 artículos potencialmente relevantes. Tras aplicar los criterios de selección, se analizaron 16. Los resultados muestran cómo en 2020 se publicaron más experiencias, abarcando a profesorado y estudiantado de grados de ramas sociales, humanidades, sanitarias y técnicas, aunque su implantación es menor que países culturalmente relacionados. Se ha estudiado la gamificación sola o asociada a otras metodologías como flipped classroom, estudiando los efectos sobre la motivación mediante encuestas y cuestionarios, demostrando efectos positivos sobre el aprendizaje y rendimiento académico.

Palabras claves: Educación Superior; Gamificación; Motivación; España. 


\begin{abstract}
Gamification is gaining momentum as an educational innovation to motivate and engage students in their learning. The digital turn in Spanish education manifests the problem of motivation, commitment and enjoyment of students, but so far, there is no research that reveals the role of gamification in university studies carried out in Spain, since this methodology has been developed Recently. Our research carries out an analysis of experiences in higher education in Spain in recent years. Part of a systematic mapping design of articles in 'Web of Science', with the following inclusion criteria: empirical gamification studies established in Spain, published between 2016 and 2020 and carried out in real environments. The initial search returned 332 potentially relevant articles. After applying the selection criteria, 16 were analyzed. The results show how more experiences were published in 2020, encompassing teachers and undergraduate students from social, humanities, health and technical branches, although their implementation is less than that of culturally related countries. Gamification has been studied alone or in association with other methodologies such as flipped classroom, studying the effects on motivation through surveys and questionnaires, demonstrating positive effects on learning and academic performance.
\end{abstract}

Keywords: Higher Education; Gamification; Motivation; Spain.

Resumo: A gamificação está ganhando força como uma inovação educacional para motivar e envolver os alunos em seu aprendizado. A virada digital na educação espanhola manifesta o problema de motivação, compromisso e prazer dos alunos, mas até agora, não há pesquisas que revelem o papel da gamificação nos estudos universitários realizados na Espanha, uma vez que esta metodologia foi desenvolvida recentemente. Nossa pesquisa realiza uma análise das experiências no ensino superior na Espanha nos últimos anos. Parte de um projeto de mapeamento sistemático de artigos em 'Web of Science', com os seguintes critérios de inclusão: estudos empíricos de gamificação estabelecidos na Espanha, publicados entre 2016 e 2020 e realizados em ambientes reais. A busca inicial retornou 332 artigos potencialmente relevantes. Após a aplicação dos critérios de seleção, foram analisados 16. Os resultados mostram como em 2020 foram publicadas mais experiências, abrangendo professores e alunos de licenciaturas nas áreas social, humanas, saúde e técnicas, embora a sua implementação seja inferior à de países culturalmente relacionados. A gamificação tem sido estudada isoladamente ou em associação com outras metodologias, como a sala de aula invertida, estudando os efeitos na motivação por meio de pesquisas e questionários, demonstrando efeitos positivos na aprendizagem e no desempenho acadêmico.

Palavras-chave: Educação Superior; Gamificação; Motivação; Espanha.

Cómo citar este artículo: Alonso-García, S., Martínez-Domingo, J.A., Berral-Ortiz, B., y De la Cruz-Campos, J.C. (2021). Gamificación en Educación Superior. Revisión de experiencias realizadas en España en los últimos años. Hachetetepé. Revista científica en Educación y Comunicación, (23), 1-21. https://doi.org/10.25267/Hachetetepe.2021.i23.2205

\title{
1. INTRODUCCIÓN
}

\subsection{La gamificación como metodología de aprendizaje}

Hoy en día el cuerpo docente debe hacer frente a estudiantes que aprenden en las aulas con desmotivación. Berenguer (2016) señala que para hacer frente a este hecho y que la educación sea más estimulante para el estudiantado, son cada vez más los profesores y maestros que progresivamente se centran en hacer uso de metodologías activas en la enseñanza. Para favorecer esta línea, las instituciones educativas están experimentando una transformación innovadora en la última década. El aprendizaje se 
está viendo influido por el empleo de plataformas o redes sociales, así como por el uso del juego dentro del proceso de enseñanza-aprendizaje (E-A). En la actualidad, la metodología de aprendizaje basada en juegos (gamificación) está experimentando un aumento de puesta en práctica en la enseñanza. Esta es una metodología centrada en hacer uso de diseños o elementos vinculados al juego en contextos de aprendizaje (Attali y Arieli-Attali, 2015). En este contexto, Wiggins (2016) sostiene que, si bien el término gamificación es novedoso, el enfoque no lo es. Según sus resultados, las estrategias de gamificación actuales parecen ser un reempaquetado de las estrategias de instrucción tradicionales. El aprendizaje basado en la gamificación ha cambiado los roles del profesorado, ha mejorado la participación del alumnado de manera más efectiva a sus intereses actuales al tiempo que ha favorecido la alfabetización digital y promovido una educación de calidad y sostenible (Haruna et al., 2018).

A este cambio también se está uniendo la Enseñanza Superior, adecuando la formación del estudiantado universitario a estas transformaciones en el aprendizaje (Gómez y Ruiz, 2017). Para ello, se han incluido plataformas de aprendizaje de predominio digital que han mejorado la percepción de los estudiantes de ciertos conceptos en la enseñanza, que han aumentado su participación en las lecciones y que les ha motivado para aprender en un entorno más interactivo y estimulante (Campillo-Ferrer et al., 2020).

\subsection{Conceptualización de gamificación}

La definición más concreta del término, y una de las más extendidas en los artículos de esta temática, es la dada por Werbach y Hunter (2014) que definen gamificación como "el uso de elementos de juegos y técnicas de diseño de juegos en contextos que no son de juegos".

Por su parte, Teixes (2014) completa esta definición añadiendo además la motivación como factor importante "la gamificación es la aplicación de recursos de los juegos (diseño, dinámicas, elementos, etc.) en contextos no lúdicos para modificar comportamientos de los individuos mediante acciones sobre su motivación".

Según Kapp (2012, p.9) "la gamificación es el uso de las mecánicas basadas en el juego, la estética y el pensamiento de juego para involucrar a las personas, motivar la acción, promover el aprendizaje y resolver problemas".

Aplicada al ámbito educativo la definición de Hernández-Padrón (2018) es la siguiente "gamificar en un contexto educativo, implica partir del currículo y realizar una propuesta didáctica utilizando el funcionamiento y la mecánica del juego, y así aprovechar sus ventajas como elemento motivador, social e interactivo" (p.154)

Lee y Hammer (2011) entienden la gamificación como una oportunidad mediante la que se pueden solventar dificultades en educación, como es el compromiso del estudiantado o la motivación del mismo. Además, destaca porque son los estudiantes los que aprenden mediante ejercicios vinculados al juego, adquiriendo una formación significativa, siendo el alumnado el protagonista de su aprendizaje (Marín-Díaz, 2015). A esto se añade que, al hacerse uso de la tecnología, esta forma de enseñanza es más atractiva para el alumnado a la hora de aprender (Gay y Burbridge, 2016).

Así pues, concretamente en educación superior, la gamificación incentiva a que se lleven a cabo actividades vinculadas al juego que son disfrutables por parte de los estudiantes, a través de las que se adquiere conocimiento, siendo este aprendido de forma divertida, teniendo la intención de que el alumnado se enfoque de una forma más activa e-ISSN:2172-7910

Doi: 10.25267/Hachetetepe.2021.i23.2205

Universidad de Cádiz 
en el proceso de E-A. Además, no se centra en la equivocación de los estudiantes cuando aprenden, siendo los errores entendidos como oportunidades en la formación. El estudiante ahora es un jugador que aprende a través de actividades gamificadas, por lo que aumenta el compromiso del alumnado con su aprendizaje, debido a tener que superar retos para aprender (Ardila-Muñoz, 2019).

Analizando estas definiciones de gamificación se observa que gamificar no consiste en jugar sin más, la gamificación persigue emplear los recursos propios de los juegos para potenciar la motivación, lo que en educación puede resultar de gran utilidad, pues el juego es una actividad ampliamente relacionada con la diversión en edades escolares y formativas.

Lo que se persigue con este aumento de la motivación es mejorar la predisposición del alumnado en el aula y optimizar así el proceso de E-A.

\subsubsection{Motivación y gamificación}

Uno de los motivos por el cual muchos docentes utilizan la gamificación como metodología, es su estrecha relación con la motivación. Tal y como indica Teixes (2014) "la gamificación se fundamenta en la capacidad que sus sistemas tienen para estimular la motivación de los usuarios/jugadores a que desarrollen unas conductas o actividades concretas".

Según Ryan y Deci (2000) se distinguen principalmente dos tipos de motivación: la intrínseca y la extrínseca.

La motivación intrínseca es la que surge del propio individuo para satisfacer sus necesidades psicológicas. Según la Teoría de Autodeterminación de Deci y Ryan (1985), estas necesidades son tres:

- Autonomía.

- Competencia.

- Relación.

La motivación extrínseca es la que surge motivada por la obtención de recompensas y consecuencias externas al propio individuo. Zarzycka-Piskorz (2016) subrayó que la educación ludificada se basa en la motivación, especialmente la motivación intrínseca que fomenta el compromiso de un individuo. Los enfoques de aprendizaje basados en juegos aumentan el interés de los alumnos en el tema porque disfrutan (o experimentan placer) a medida que aprenden y, por lo tanto, están más comprometidos y enfocados en el tema. Lo idóneo en el proceso de gamificación es que los alumnos tengan motivación intrínseca, pero esto no es fácil, por lo que los docentes nos podemos valer de la motivación extrínseca como herramienta para conseguir la motivación intrínseca.

Otra de las teorías que pueden reforzar el empleo de la gamificación como herramienta para potenciar la motivación es la "Teoría del Flujo", una teoría del psicólogo Mihaly Csíkszentmihalyi. Según Csíkszentmihalyi (1997) el flujo ("flow" en inglés) es un "estado óptimo en el que la concentración activa y la absorción en la actividad creativa conducen a la sensación de tiempo completamente suspendido", la persona que alcanza este estado con la realización de alguna actividad se implica de manera completa y disfruta llevándola a cabo. Este estado mental es el que se consigue cuando jugamos, ya que en muchos casos estamos tan inmersos que se pierde la noción del tiempo. Fernández Solo (2014) además plantea que la gamificación aumenta la motivación a nivel neuronal ya que jugando se produce dopamina, el neurotransmisor encargado de regular la e-ISSN:2172-7910

Doi: 10.25267/Hachetetepe.2021.i23.2205

Universidad de Cádiz 
motivación. Todo ello refuerza el empleo de la gamificación como metodología activa que pretende motivar al alumnado en su proceso de E-A.

\subsubsection{Fases del diseño de un sistema gamificado}

A la hora de implementar un proceso gamificado, Werbach y Hunter (2014), establecen 6 pasos:

- Definir los objetivos.

- Delinear los comportamientos objetivos.

- Describir a los jugadores.

- Desarrollar ciclos de actividad.

- ¡No olvidarse de la diversión!

- Implantar las herramientas apropiadas.

Por su parte, Teixes (2014) analiza modelos de diseño propuestos por distintos autores de la materia, entre otros el de Werbach y Hunter (2014), elaborando el suyo propio, en el que hace especial hincapié en la importancia del inicio del proceso y en especial a:

- Los objetivos a conseguir.

- Por qué aplicar la gamificación.

El modelo resultante elaborado por Teixes (2014) se muestra en la figura 1.

\section{Figura 1}

Rectángulo redondeado: Definir los objetivos

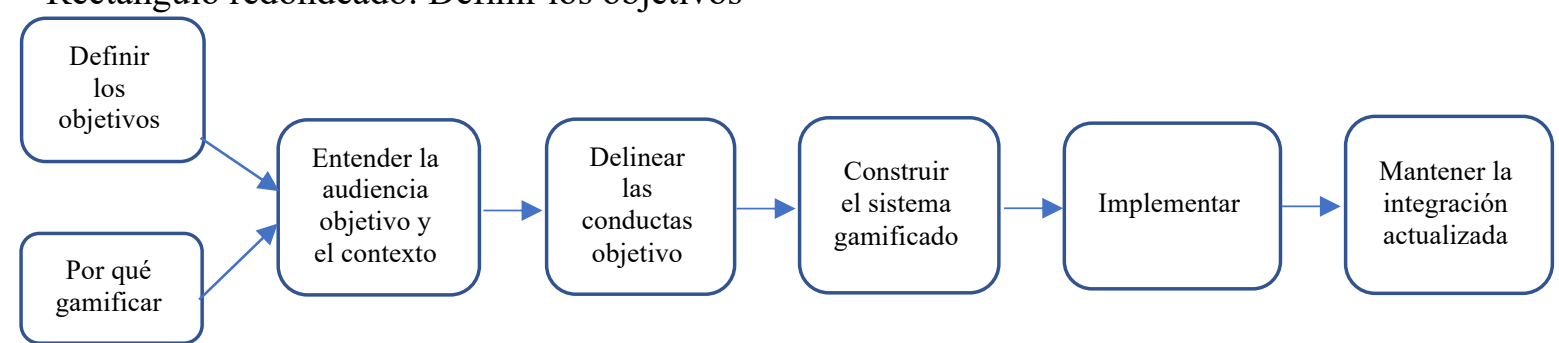

Fuente: Teixes (2014).

\subsubsection{La gamificación en España}

Para poder llevar a cabo este tipo de metodologías, los docentes necesitan una formación con el objetivo de trabajar de forma correcta dentro del aula de forma didáctica. Por tanto, todo docente que ponga en práctica esta metodología, debe tener las capacidades necesarias para desarrollar las actitudes correctas en los estudiantes, evitando el fomento de la competitividad y el protagonismo entre el alumnado, por lo que, debe incrementarse el valor del grupo y un entorno en el que los estudiantes participen y se comprometan de forma activa (Rovira-Collado et al., 2016). En este sentido, la realidad educativa española está avanzando hacia un aprendizaje apoyado en recursos tecnológicos debido a múltiples factores, entre los que destacan que España lidera el uso de teléfonos inteligentes dentro de la Unión Europea ( $81 \%$, respecto a un $71 \%$ de media en la UE), así como en la oferta de Cursos online masivos y abiertos (MOOC, por sus siglas en inglés), que representa el $28,2 \%$ de la toda la oferta de la UE en 2015, la disponibilidad de conexiones en banda ancha y fibra óptica, y desde hace 15 años, las universidades españolas han desarrollado gestores electrónicos que permiten el 
aprendizaje online (plataformas Moodle, LRN, Sakai). Así mismo, España ha sido uno de los países que mejor se ha adaptado al súbito cambio de docencia presencial a virtual durante la pandemia Covid-19, aunque se carece de una visión estratégica sobre la transformación digital de la educación superior para los tiempos post Covid-19 (AreaMoreira et al., 2021).

El objetivo de este trabajo es recopilar las últimas experiencias en gamificación llevadas a cabo en España y extraer los elementos básicos que sustentan el proceso de EA basado en la gamificación. En el mismo analizaremos sistemáticamente los artículos a los que se accede a través de una búsqueda en la base de datos Web of Science con palabras clave seleccionadas y realizaremos una discusión con otros conceptos básicos relacionados.

\section{METODOLOGÍA}

El estudio se ha realizado mediante análisis de documentos y análisis de contenidos relacionados desde 2016 a 2020. El proceso básico ha consistido en recopilar datos similares en el marco de la gamificación y temas mencionados e interpretarlos de manera uniforme. El análisis de contenido es un marco científico que permite un análisis sistemático de escritos, orales y otras fuentes (Krippendorff, 2018).

Para conocer el trabajo de los profesores universitarios españoles en el campo de la gamificación, se examinaron los documentos de Web of Science, con las palabras clave "High Education" o sus términos relacionados según el Tesauro, "Gamificación" y "España", entre los años 2016 y 2020. Se seleccionaron aquellos publicados por autores españoles o en instituciones españolas, en revistas revisadas por pares, en español o inglés y que recogían experiencias prácticas de gamificación. No se incluyeron revisiones.

\section{RESULTADOS}

La búsqueda arrojó 337 documentos de los cuales se seleccionaron 16 —Figura 2 - 


\section{Hachetetepé. Revista científica de Educación y Comunicación}

$\mathrm{n}^{\mathrm{0}} 23,1-21,2021$

\section{Figura 2}

Diagrama de flujo acorde a la declaración PRISMA, 2009.
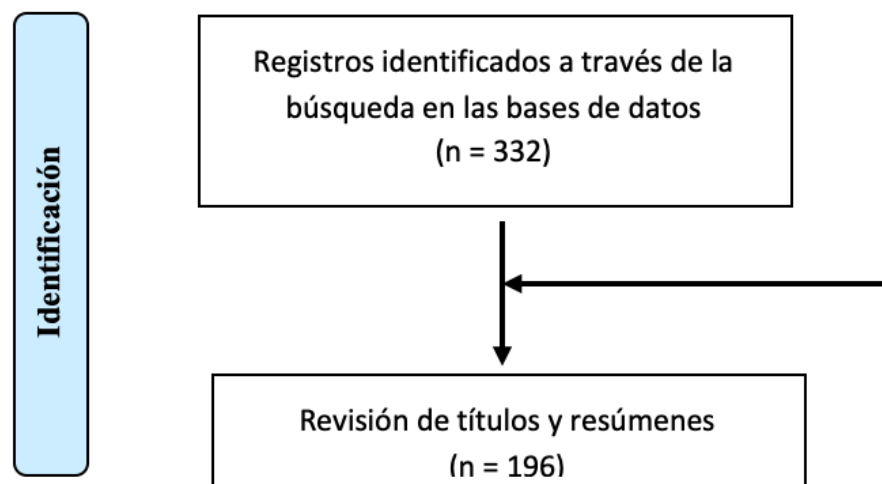

Registros incluidos tras revisar referencias $(n=5)$

Revisión de títulos y resúmenes ( $n=196$ )
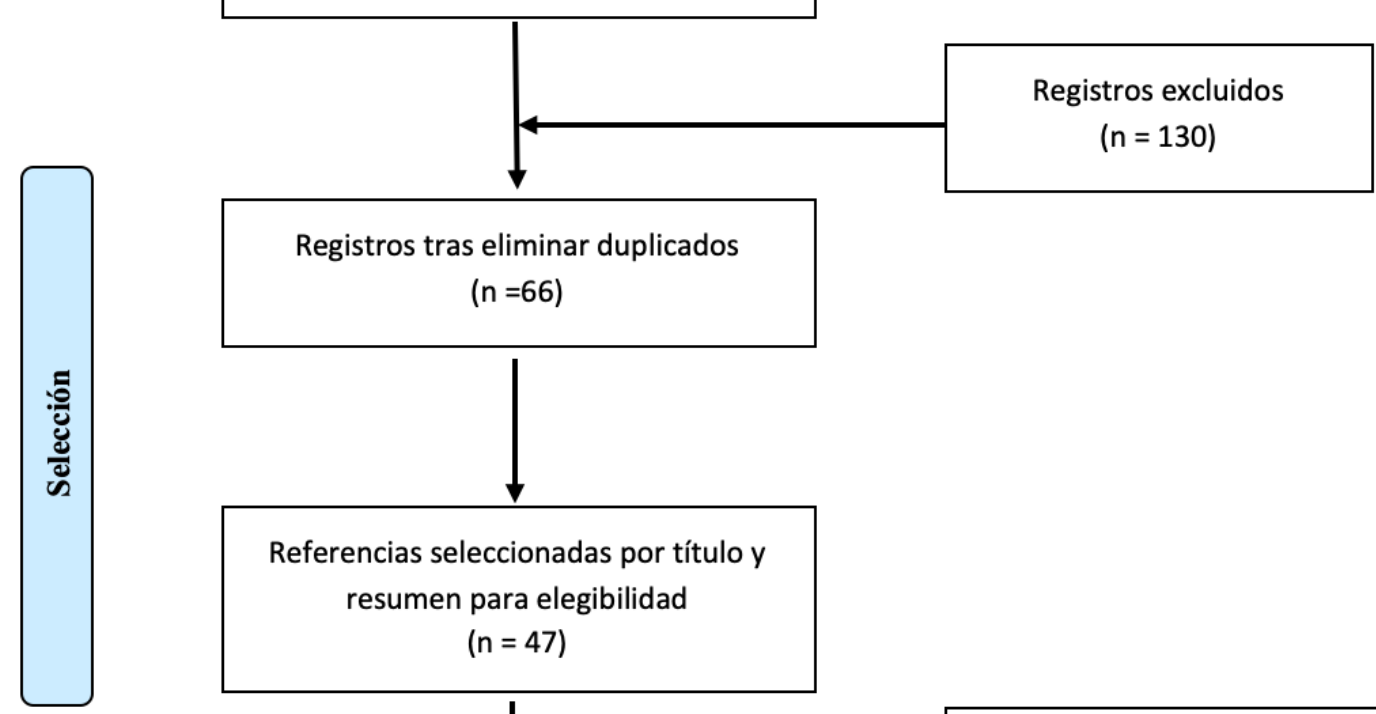

Registros tras eliminar duplicados ( $n=66$ )

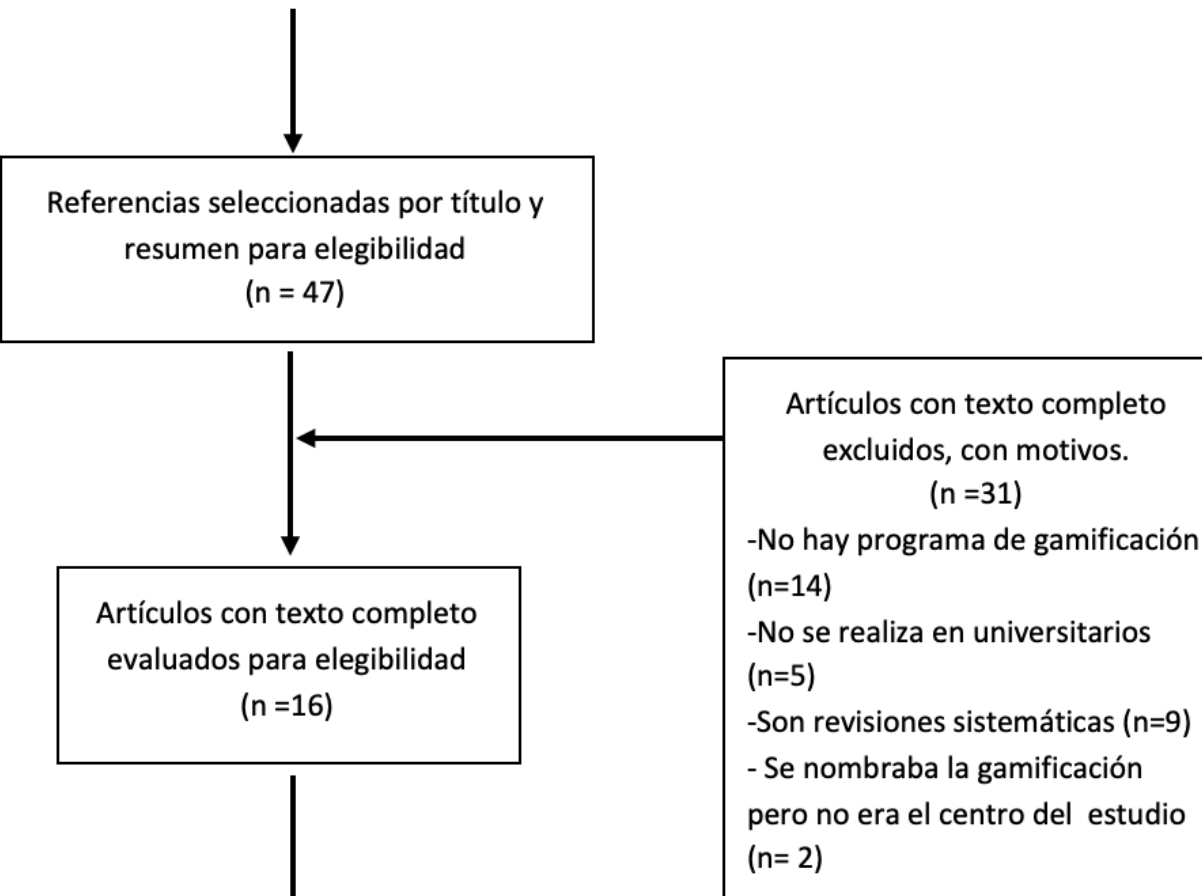

Estudios incluidos en síntesis cualitativa.

$(n=16)$ 
Los documentos seleccionados han sido analizados, integrados por correlación entre sí y los datos se analizaron mediante análisis de contenido. Cohen y Manion (2001) describen el análisis de contenido como una presentación de los mensajes contenidos en los documentos seleccionados de manera concisa - Tabla 1 -

Tabla 1

Análisis de contenido

\begin{tabular}{|c|c|c|c|c|c|c|}
\hline AUTOR & OBJETIVO & PARTICIPANTES & $\begin{array}{l}\text { TIPO DE } \\
\text { ESTUDIO }\end{array}$ & $\begin{array}{c}\text { HERRAMIENTA } \\
\text { UTILIZADA }\end{array}$ & RESULTADOS & CONCLUSIONES \\
\hline $\begin{array}{l}\text { Cózar- } \\
\text { Gutiérrez y } \\
\text { Sáez-López } \\
(2016) . \\
\text { España. } \\
1 \text { centro. }\end{array}$ & $\begin{array}{l}\text { Promover una } \\
\text { adecuada } \\
\text { formación inicial } \\
\text { en TIC de los } \\
\text { futuros profesores } \\
\text { de Educación } \\
\text { Primaria. }\end{array}$ & $\begin{array}{l}89 \text { estudiantes }(75.3 \\
\% \mathrm{~F} ; 24.7 \% \mathrm{~V}) \text { de } 2^{\circ} \\
\text { curso del grado de } \\
\text { Educación Primaria } \\
\text { matriculados en } \\
\text { Ciencias Sociales II: } \\
\text { asignatura Historia y } \\
\text { Docencia. }\end{array}$ & 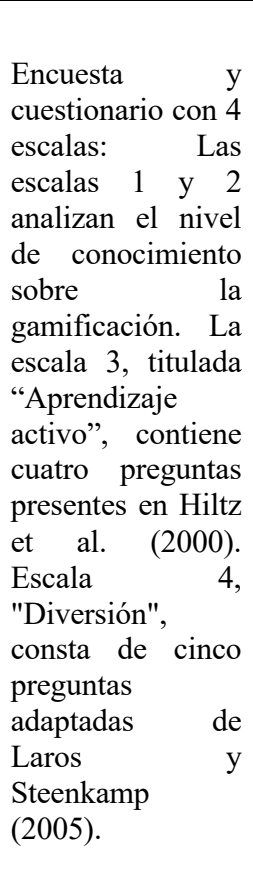 & $\begin{array}{lr}\text { MinecraftEdu } & \text { de } \\
\text { Sáez-López } & y \\
\text { Domínguez } & (2013) \\
\text { Juego } & 3 \mathrm{D} \\
\text { interactivo. } & \end{array}$ & 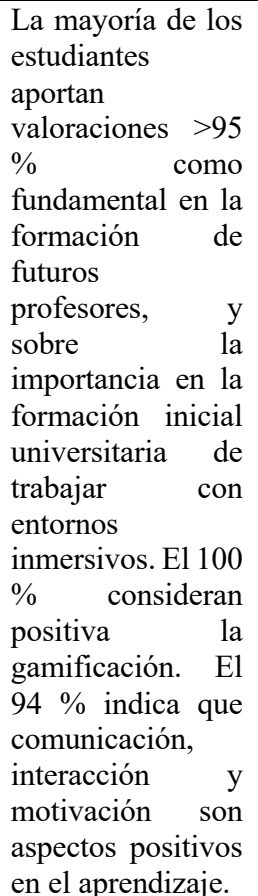 & $\begin{array}{l}\text { Proporciona } \\
\text { evidencia de } \\
\text { actitudes, valores y } \\
\text { perspectivas sobre } \\
\text { el aprendizaje } \\
\text { basado en juegos y } \\
\text { la gamificación en } \\
\text { entornos } \\
\text { universitarios. } \\
\text { MinecraftEdu } \\
\text { beneficia aspectos } \\
\text { pedagógicos que } \\
\text { permitan una mayor } \\
\text { actividad, } \\
\text { motivación } \\
\text { implicación de los } \\
\text { estudiantes. }\end{array}$ \\
\hline $\begin{array}{l}\text { Sánchez.Mena } \\
\text { y Martín- } \\
\text { Parreño. } \\
\text { (2016). } \\
\text { España. } \\
1 \text { centro. }\end{array}$ & $\begin{array}{lr}\begin{array}{l}\text { Analizar } \\
\text { principales }\end{array} & \text { las } \\
\text { barreras } & \text { e } \\
\text { impulsos } & \text { para } \\
\text { alentar a } & \text { los } \\
\text { profesores } & \\
\text { universitarios para } \\
\text { utilizar } \\
\text { herramientas } \\
\text { gamificación. de }\end{array}$ & $\begin{array}{l}26 \quad \text { profesores } \\
\text { universitarios (Edad, } \\
\text { media: 43,78; Rango: } \\
\text { 26-65) que utilizan la } \\
\text { gamificación en su } \\
\text { actividad docente }\end{array}$ & $\begin{array}{l}\text { Aproximación } \\
\text { fenomenológica } \\
\text { mediante } \\
\text { entrevistas } \\
\text { estructuradas. } \\
\text { Análisis de texto } \\
\text { utilizando la } \\
\text { herramienta } \\
\text { WordStats } 7.0 .11 \\
\text { RQ1. }\end{array}$ & Ninguna. & $\begin{array}{l}\text { Las respuestas } \\
\text { más utilizadas } \\
\text { estaban en } \\
\text { relación con la } \\
\text { motivación, } \\
\text { entretenimiento, } \\
\text { atención - } \\
\text { motivación } \\
\text { interacción. }\end{array}$ & $\begin{array}{lrr}\text { La gamificación } \\
\text { puede ser útil para } \\
\text { estimular a } r \text { los } \\
\text { alumnos para la } \\
\text { participación } & y \\
\text { motivación. } & \end{array}$ \\
\hline $\begin{array}{l}\text { Borras-Gene } \\
\text { et al. } \\
(2016) . \\
\text { España. } \\
2 \text { centros. }\end{array}$ & $\begin{array}{l}\text { Determinar si la } \\
\text { incorporación de } \\
\text { metodologías y } \\
\text { estrategias de } \\
\text { gamificación en } \\
\text { Cursos online } \\
\text { open masivos } \\
\text { puede potenciar la } \\
\text { motivación de los } \\
\text { participantes y } \\
\text { puede incidir en la } \\
\text { participación, } \\
\text { compromiso y } \\
\text { fidelización de los } \\
\text { estudiantes. }\end{array}$ & $\begin{array}{lr}60 \text { estudiantes } & \text { o } \\
\text { licenciados } & \text { en } \\
\text { ingeniería. } & \end{array}$ & $\begin{array}{l}\text { Encuestas online } \\
\text { mediante } \\
\text { entrevistas semi } \\
\text { estructuradas. } \\
\text { Diseño } \\
\text { explicativo } \\
\text { secuencial de } \\
\text { métodos mixtos, } \\
\text { que integra los } \\
\text { métodos } \\
\text { cuantitativos y } \\
\text { cualitativos } \\
\text { durante la fase de } \\
\text { interpretación. }\end{array}$ & $\begin{array}{l}\text { Google }+ \text {, } \\
\text { Hangouts } \\
\text { Instagram, } \\
\text { Certificados } \\
\text { insignias. }\end{array}$ & 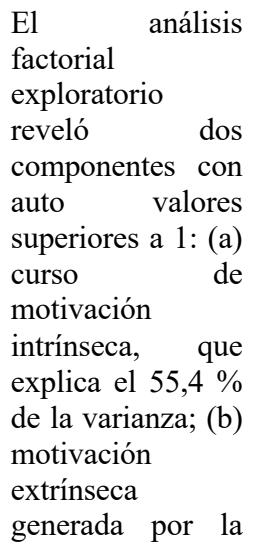 & $\begin{array}{l}\text { La mayoría de los } \\
\text { estudiantes son } \\
\text { positivos sobre la } \\
\text { gamificación y el } \\
\text { uso de las redes } \\
\text { sociales en } \\
\text { educación la } \\
\text { especialmente } \\
\text { los cursos on } \\
\text { online. }\end{array}$ \\
\hline
\end{tabular}




\section{Hachetetepé. Revista científica de Educación y Comunicación}

$\mathrm{n}^{\mathrm{0}} 23,1-21,2021$

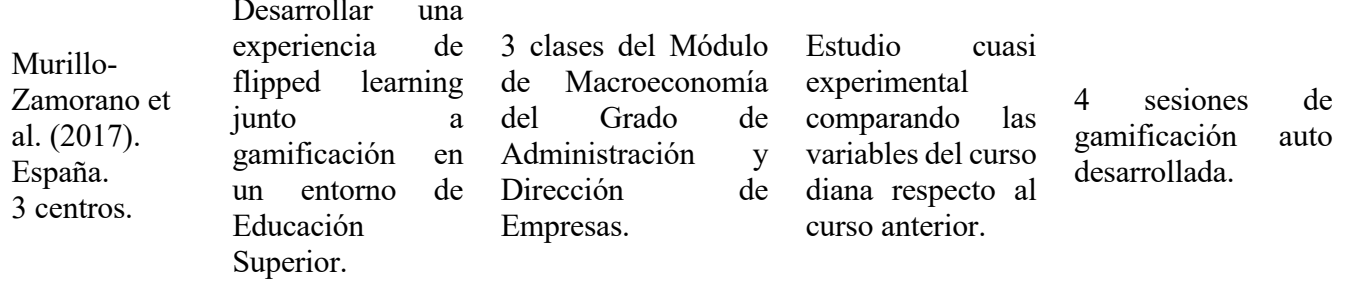

Superior.
Galbis-

Córdoba, et al. (2017). España.

1 centro.
Ares et al. (2018). España.

\section{Desarrollar y probar \\ empíricamente un modelo de investigación de los factores que contribuyen a la actitud de los estudiantes hacia el uso de videojuegos educativos en línea diseñados para desarrollar sus competencias.}

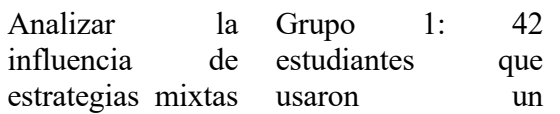

Diseño

cuantitativo,

mediante

cuestionarios

autoadministrados

Se estimó el

modelo teórico

mediante el

algoritmo Partial

Least Squares

(PLS) a través de

SmartPLS $\quad 3.0$

(Ringle et al.,

$\begin{array}{lrlr}\text { Muestra } & \text { de } & \text { 2014). } \\ \text { conveniencia. } & 128 & \text { atención percibida }\end{array}$

estudiantes $(48 \% \mathrm{~V}$; se midió

$42 \% \mathrm{~F}$; (Rango de adaptando cuatro edad=17-41 años) de elementos de $\mathrm{Su}$ universidades \& Cheng (2015).

privadas de grados Se adaptaron sanitarios cuatro elementos

(fisioterapia, de (Ibidem) para

odontología),

medir la

sociales (marketing,

criminología,

psicología, derecho,

lengua,

comunicación y adaptando cuatro

relaciones sociales) y elementos de

técnicos (Ibidem). La

(arquitectura). actitud hacia los

videojuegos

educativos en

línea se midió

adaptando tres

elementos de

Chattopadhyay y

Basu (1990). Los

ítems del

cuestionario se

midieron

utilizando una escala tipo Likert de 5 puntos

\section{Metodología}

estrategias mixtas

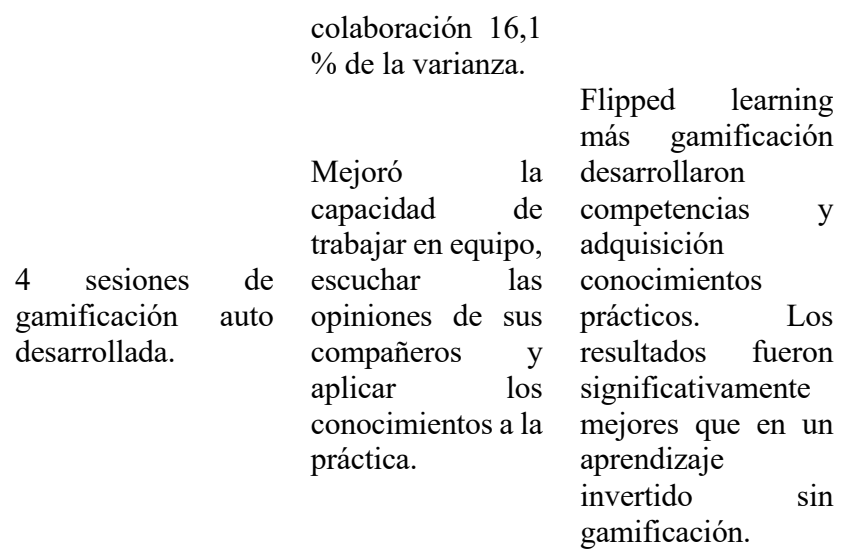

La atención

Las expectativas de los estudiantes respecto a los videojuegos percibida, diseñados para percepción, desarrollar sus confianza y competencias, se relevancia centran en tres percibida, áreas: a) deben contribuyen contener

positivamente a la características que actitud de los llamen la atención estudiantes hacia de los estudiantes, el uso de b) deben ser videojuegos equilibrados, educativos en teniendo en cuenta línea diseñados el nivel de dificultad para desarrollar y las competencias sus competencias. de juego de los estudiantes, c) deben ser percibidos como relevantes por los estudiantes.

Plataforma cuestionarios Kahoot! y El uso de Kahoot! dio lugar a un aumento
En un aula universitaria, Kahoot! mejoró en 


\section{Hachetetepé. Revista científica de Educación y Comunicación}

$\mathrm{n}^{\mathrm{0}} 23,1-21,2021$

2 centros

en el aprendizaje de química a nivel universitario y el grado desarrollo del conocimiento de los estudiantes.
Gómez-

carrasco et al. (2019).

España

1 centro.
Cuevas-

Martínez et al. (2019).

España.

2 centros. cuestionario Kahoot! por cada clase recibida en el grado de Química

Grupo 2: 47 estudiantes que usaron el mismo cuestionario cada dos clases. de estudiantes con diferente

frecuencia de uso. Se comparaban Los resultados académicos de estos dos grupos de

alumnos y estos a su vez con los obtenidos el año anterior, en el que Kahoot! no fue empleado.
210 estudiantes (53

Establecer el V, $25 \%$; $157 \mathrm{~F}, 75$ impacto en la \%). (90\% de entre motivación y percepción del aprendizaje logrado a través de estrategias y técnicas asociadas a gamma y flipped classroom.

Pertenecientes a cuatro grupos de la asignatura Metodología didáctica para la Docencia de las Ciencias Sociales en
Estudio

descriptivo

cuantitativo con Flipped classroom Selección de como enfoque de muestra no probabilística el Grado de Educación Primaria

99 alumnos (Edad media: 23,$9 ; \quad \mathrm{DS}=$

Determinar la 7,9) del 3 curso del influencia de una grado de Ingeniería propuesta de de gamificación en el rendimiento académico.

usando un
cuestionario ad hoc "Evaluación del programa formativo basado en gamificación y aula invertida", del tipo Likert cerrado.

Estudio cuasi experimental con medidas únicas durante 4 cursos académicos comparando los resultados académicos durante el curso experimental en midieron:

Desconexión:

porcentaje de tareas que no fueron entregadas.

- Efectividad: porcentaje de tareas superadas entre todas las tareas
Telecomunicaciones en una asignatura obligatoria y dos optativas durante 4 cursos académicos
Cada cuestionario constaba de 7 preguntas y ofrecía cuatro respuestas diferentes con una única opción correcta, respondida mediante

el

Smartphone de cada estudiante Tiempo de respuesta: 30 segundos para responder cada pregunta.

Finalmente, el profesor daba una breve explicación después de cada pregunta. relación a las calificaciones obtenidas cursos previos.

Estudio

Analizar $\quad$ los 210 alumnos (53 V; efectos de un $25 \%$ y $157 \mathrm{~F} ; 75 \%$ ) programa de (Edad media: 20.94;

Gómezformación basado $\mathrm{DS}=2.77$; rango: 19 -

Carrasco et al. (2020).

España.

2 centros.

Saga Súper Mario. evaluadas.

- Rendimiento: nota media de todas las tareas evaluadas de 0 a 10

Flipped classroom y gamificación siguiendo Teixes.

\section{las} en flipped classroom y la gamificación

sobre

44) de 4 grupos del Grado de Educación Primaria. Asignatura; Metodologia de la aprendizaje y los enseñanza de las ciencias sociales. descriptivo

cuantitativo. El aprendizaje logrado a través del programa se evaluó mediante la recolección de percepciones a través de un

significativo en las calificaciones generales $\mathrm{y}$ en el número de estudiantes que aprobaron la asignatura.

Además, también se observaron algunas

diferencias en el rendimiento

académico de los estudiantes según el grupo.

Hubo efectos positivos sobre la motivación, tanto extrínseca como intrínseca y mayor puntuación de la motivación intrínseca, especialmente la relacionada con las estrategias de enseñanza

utilizadas sobre la extrínseca, donde el mero hecho de aprobar se puntuó más bajo.

En cada sujeto se Los alumnos se

sienten cómodos con las reglas e intentaron mejorar su puntuación para vencer a los demás. Esto eventualmente significa que obtuvieron mejores notas y la aceptación del experimento de gamificación.

\section{Estadísticas} descriptivas; pruebas de medias ( $t$ de Student y ANOVA de un factor); pruebas no paramétricas (prueba de UMann Whitney); y correlaciones de

general el aprendizaje y las calificaciones de los estudiantes, y que esta mejora era más prevalente entre los estudiantes que habían logrado un mejor rendimiento en Kahoot!

La aplicación del programa de formación basado en la gamificación y en flipped classroom tuvo un efecto positivo en la motivación $y$ percepción de aprendizaje de los estudiantes.

La aplicación de la gamificación fue positiva en general para el proceso de aprendizaje de todos los estudiantes que participaron y no supuso ninguna 


\section{Hachetetepé. Revista científica de Educación y Comunicación \\ $\mathrm{n}^{\mathrm{0}} 23,1-21,2021$}

resultados de los Duración docentes en semestre. formación. un

cuestionario ad hoc y una escala de observación de las unidades didácticas diseñadas.
En qué medida la participación del alumnado en una plataforma de juegos en línea puede promover el nivel, el tipo de participación, la motivación, el compromiso y el aprendizaje significativo.
Desarrollar la capacidad de los educadores universitarios para trabajar con prácticas

García-

Holgado et al.

(2020).

España.

2 centros.

Portugal,

Irlanda

Alemania.

Torres-

Toukoumidis,

España.

México.

Ecuador. et al. (2020).

1 centro. educativas

abiertas que abarquen el uso y la producción de recursos educativos

abiertos, aplicación de pedagogías abiertas y la evaluación de los resultados del aprendizaje.

Explorar el conocimiento teórico y práctico del profesorado universitario

sobre gamificación y sus aplicaciones didácticas, así como diferenciación entre
Muestra de conveniencia de 101 estudiantes del $2^{\circ}$ curso del grado de Educación Primaria (unidad obligatoria, "Enseñanza de las Ciencias Sociales") ( $25 \mathrm{~V} ; 24.7 \%$. $76 \mathrm{~F}$, $75.3 \%$ ) Edad media: 20,94; $\quad \mathrm{DS}=2,77$; Rango: 19 y 22 años.
Cuasi

Experimental con cuestionarios pre y post con una metodología cuantitativa.

Toma de datos mediante

cuestionario ad hoc.

$\begin{array}{ll}\text { Consorcio formado 6 } & \begin{array}{l}\text { Manual de } \\ \text { prácticas de } \\ \text { enseñanza abierta, }\end{array} \\ \text { universidades } & \begin{array}{l}\text { un Plan de } \\ \text { europeas, y 5 }\end{array} \\ \begin{array}{l}\text { Hispanoamericanas } \\ \text { con experiencia en } \\ \text { de aprendizaje }\end{array} \\ \begin{array}{l}\text { onliencia esquemas } \\ \text { de educación acreditación } \\ \text { abierta. }\end{array} \\ \begin{array}{l}\text { abiertos y un Kit } \\ \text { OpenGame. }\end{array}\end{array}$
OpenGame.
Plataforma Kahoot.

Se aumentó la motivación tanto en varones como en mujeres, el nivel competencia digital y actitudes generales, así como mayor satisfacción en el desarrollo de sus competencias sociales y cívicas.

\section{Proyecto}

OpenGame con un manual que proporcionaba un conjunto de ejemplos que los educadores pueden adaptar y replicar en diferentes contextos.

85 profesores universitarios de 46 universidades

públicas y 39 de privadas de España, 88 de México (36, 52) y 87 de Ecuador la $(32,55)$. para escala Likert. El
Estudio

exploratorio de medida única mediante cuestionarios cerrados online. Se utilizó con una cuestionario estuvo compuesto por 4 preguntas introductorias $\mathrm{y}$ gamificación y el

Pearson entre aprendizaje fueron satisfactorios, aunque inferiores a los percibidos.

El uso de Kahoot permitió adquirir conocimientos y desarrollar un conjunto habilidades vinculadas competencias sociales y cívicas. Se sugiere que se debe alentar a los profesores $\mathrm{y}$ estudiantes a adquirir conocimientos digitales y dominar herramientas TIC, para fomentar la creatividad y el pensamiento crítico, al tiempo que amplía su repertorio de enseñanza. y habilidades de aprendizaje

El proyecto

Diseño de un OpenGame $\begin{array}{llr}\text { currículo } & \text { incrementa } & \text { la } \\ \text { educativo basado } & \text { motivación de los }\end{array}$ en la gamificación educadores a través un aprendizaje de la gamificación educativo en línea adquiriendo las competencias de entrenamiento de educación abierta profesorado tanto online como al universitario y un aire libre

esquema de y complementa acreditación. el marco DigCompEdu.

Ninguna.

El $65 \%$ de los La aplicación profesores universitarios conoce $62 \%$ identifica los elementos del juego. El $43 \%$ reconoce existencia de aspectos psicológicos práctica de la gamificación en enseñanza superior es menor en España que en México y Ecuador, así como en Universidades privadas que en las públicas. significativamente 


\section{Hachetetepé. Revista científica de Educación y Comunicación}

$\mathrm{n}^{\mathrm{0}} 23,1-21,2021$

universidades

públicas

privadas.

\begin{abstract}
15 preguntas sobre el tema, esta última dividida en 5 preguntas orientadas a la dimensión teórica y 10 orientadas a la dimensión práctica.
\end{abstract}

Determinar si el diseño de análisis gráfico adaptado a la arquitectónica ayuda a la comprensión de conceptos teóricos
FernándezAntolín et al. (2020). España. 1 centro.
Santos-

Villalba et al.

(2020)

España

4 centros relevantes para las materias técnicas $\mathrm{y}$

determinar si el

materiales

didácticos

innovadores

influye positiva y

significativamente

sobre la

motivación del

aprendizaje de los estudiantes.

Examinar las evaluaciones de educación superior de la Universidad de Málaga sobre el diseño de la gamificación educativa y los Criterios

\section{didácticos} su efecto en la motivación del alumno, las aplicaciones de la gamificación y la diseño de los estudiantes de

relacionados con la gamificación y
171 estudiantes de arquitectura.

e

\section{Diseño}

cuantitativo,

mediante

cuestionarios

autoadministrados online.
187 estudiantes del grado de Educación Primaria.

(138 F; $73.8 \%$; $49 \mathrm{~V}$, (26.2 \%). Edad media: 22.97 (rango: 18 y 43 años).
Estudio

descriptivo

cuantitativo,

mediante

cuestionarios auto administrados

online y escala de Likert.

Se calculó el modelado de ecuaciones

estructurales de mínimos cuadrados parciales para estos datos con base en un modelo exploratorioconfirmatorio $y$ básicos

(emocionales, cognitivos conductuales). El $87 \%$ de los docentes en Ecuador, el $78 \%$ en España y el 89 $\%$ en México han aplicado la gamificación en clase. El $69 \quad \%$ reconoce los tipos de acciones docentes para gestionar el aula, pero solo el $50 \%$ saben gestionar un ranking del comportamiento de los estudiantes.

El 95,2 \% de los Herramientas de alumnos considera simulación de imprescindible construcción rendimiento.

simular un edificio a medida que lo diseñan.
El diseño de material didáctico innovador influye significativamente en la motivación de los estudiantes $\mathrm{y}$, por tanto, en su proceso de aprendizaje.
Se encontró una relación positiva entre Plataforma Moodle. evaluación de la motivación de los estudiantes y la actitud de sostenibilidad.
Las opiniones de los estudiantes sobre el papel de su material de diseño de formación $\mathrm{y}$ enseñanza en la educación afectarían la motivación de los estudiantes y el comportamiento de sostenibilidad, aunque el poder de este último constructo moderado. 


\section{Hachetetepé. Revista científica de Educación y Comunicación}

$\mathrm{n}^{\mathrm{0}} 23,1-21,2021$

actitud hacia la sostenibilidad.
Ferriz-Valero et al. (2020).

2 centros. Noruega.
Bullón y

Galloso

(2020).

España.

2 centros.

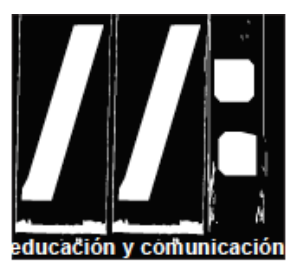

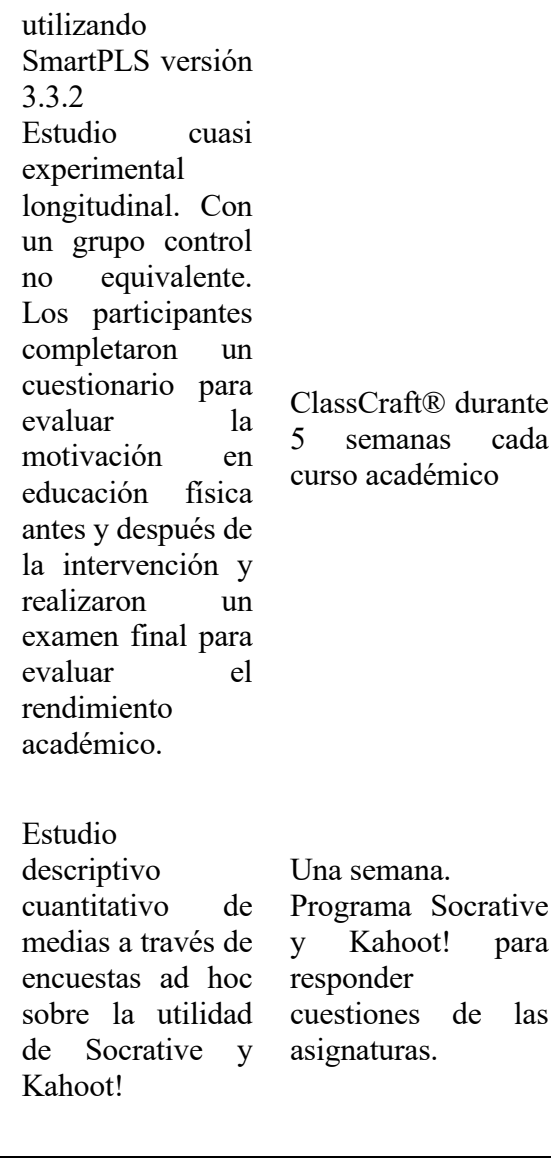

127 estudiantes de Educación física en Analizar el la asignatura impacto de la "Actividad Física en gamificación en el medio natural" (73 las motivaciones y desempeños académicos de estudiantes universitarios

$\mathrm{V}, 57,5 \%$; $54 \mathrm{~F}$; 42,5

$\%$; Edad media: 22;

$\mathrm{DS}=3,5$ años) $\mathrm{y}$ durante dos cursos académicos.
$62 \quad$ grupo

65 grupo control.

Estudiantes de

Mejorar la satisfacción de los alumnos en las asignaturas de Matemáticas y Física en los grados ingeniería ciencias.

\section{Hubo un aumento en la regulación externa solo en el grupo \\ experimental. Este grupo logró un rendimiento académico significativamente mejor.}

Las respuestas
positivas o muy
positivas r son
mayoría. Solo al
considerar ri
Socrativer o
Kahoot! les
ayudan a asistir a
clase ambos
grupos se sienten
indiferentes.

Se sugiere que la implementación gamificada es beneficiosa para el rendimiento académico en la etapa universitaria, aunque la motivación intrínseca no cambia. La naturaleza de las recompensas $\mathrm{o}$ castigos podría jugar un papel importante en los resultados.

Al usar Socrative o Kahoot!, los estudiantes se ven obligados a responder, lo que les ayuda a desarrollar las habilidades $\mathrm{y}$ competencias asociadas.

El mayor número de trabajos seleccionados han sido de $2020(\mathrm{n}=8)$. Destacan tres trabajos realizados en colaboración con instituciones de otros países, todos ellos en 2020 (Ferriz-Valero et al.; García-Holgado et al.; Torres-Toukoumidis et al.) y que 8 han sido el resultado de colaboraciones entre 2 a 4 centros de distintas instituciones españolas.

Los objetivos más mencionados han sido determinar los efectos de la gamificación sobre la motivación del alumnado $(n=6)$, y de las flipped classroom (4) como aprendizaje electrónico. Otros ítem fueron evaluar cómo la gamificación incide sobre un aprendizaje significativo (3), si mejora el rendimiento académico (2), las competencias (1), la satisfacción (1), el nivel de conocimientos (1) y la influencia de las TIC en la formación inicial del alumnado. También hubo un artículo que se marcó como objetivo determinar las barreras detectadas por el profesorado para usar la gamificación en las enseñanzas universitarias y 2 trabajos que tuvieron como objetivo determinar el nivel de conocimientos del profesorado universitario sobre gamificación.

En 6 estudios participó alumnado de Educación Primaria (entre 87 y 210 alumnos; media: 167,9), en 5 alumnado de ciencias (ingeniería civil, ingeniería química, grado de química, telecomunicaciones y arquitectura) (entre 42 y 171 participantes; media=91), en un alumnado de Educación Física (127) y en otro participaron una miscelánea de estudiantado sanitario, marketing, criminología, derecho, lengua, comunicación y relaciones sociales (128). En uno participaron 26 profesores y en otro estudio internacional otros 260 profesores.

Las técnicas de evaluación más utilizadas fueron encuestas o cuestionarios (11), seguida de determinaciones variables específicas según los objetivos del estudio como e-ISSN:2172-7910 
variables de rendimiento académico (3) y entrevistas estructuradas (2). En 1 se evaluaron materiales de prácticas y juegos de aprendizaje.

Las herramientas de gamificación utilizadas han sido muy variadas, como Kahoot! (2), Kahoot! más Socrative (1), flipped classroom mas gamificación (4), flipped classroom mas Socrative (2) y un variado grupo de herramientas como Minecraft Edu, Google + , Hangouts, Instagram, certificados e insignias, Súper Mario, OpenGame, Moodle, ClassCraft y una herramienta de simulación y construcción (1 estudio con cada herramienta). En 3 estudios no se especificó la herramienta de gamificación utilizada.

Los estudios seleccionados mostraron efectos beneficiosos o mejoras pedagógicas (4), aumento de la motivación (5) especialmente mediante el uso de flipped classroom, open games, Moodle y en las aplicaciones de la gamificación en cursos on line. Flipped learning más gamificación desarrolló competencias y adquisición conocimientos prácticos de forma más significativa que usando solo flipped learning. Kahoot! mostró mejoras en las calificaciones del alumnado y en las competencias sociales de los mismos, así como en su asociación con Socrative mientras que ClassCraft evidenció mejoras en el rendimiento académico

\section{DISCUSIONES}

Los estudios seleccionados muestran que existen evidencias sólidas para utilizar la gamificación en el contexto educativo de enseñanza superior en España, apreciando un incremento de estudios en $2020(n=8)$, indicando el auge de estudios sobre gamificación en los últimos años, aunque desconocemos el efecto que la pandemia COVID-19 pueda tener sobre los estudios de esta temática en los próximos años. No obstante, a pesar de la indudable penetración de la gamificación, su aplicación práctica en enseñanza superior es significativamente menor en España que en México y Ecuador, usándose sin un nivel de conocimientos teóricos suficientes (Torres-Toukoumidis et al., 2020). Ya en 2011 Palazón-Pérez, et al., indicaban que la penetración del autoaprendizaje activo o el trabajo colaborativo, ambos potenciados mediante la gamificación, era limitado en las universidades españolas. Aunque esto ha mejorado, aún parece ser insuficiente por comparación con otros países.

En la mayoría de estudios el juego es percibido como un conjunto de actividades que contribuyen a aliviar las tensiones académicas y de la vida diaria en el marco universitario. Así, los profesores consideran útil la gamificación para estimular a los alumnos hacia la participación y la motivación (Sánchez-Mena y Martín-Parreño, 2016) y también consideran que la gamificación mejora la motivación de los propios educadores que, a través de proyectos innovadores como el proyecto OpenGame, pueden incrementar su propia motivación y adquirir competencias de educación abierta tanto online como al aire libre (García-Holgado et al., 2020).

Los estudios realizados con autores extranjeros (además del indicado más arriba de Torres-Toukoumidis et al., 2020) denotan la cooperación con centros de investigación como Portugal, Irlanda y Alemania, aunque en este caso, no se han discriminado los distintos grados de motivación y competencias de los profesores según su origen (GarcíaHolgado et al., 2020). Esto hubiera sido importante para conocer las limitaciones, si las hubiera, de los profesores españoles respecto a sus homónimos de oros países. El estudio de Ferriz-Valero et al., (2020) realizado con un centro de investigación en Noruega, tampoco discrimina el papel de los participantes entre uno y otro país. En los estudios realizados por varios centros de investigación españoles, parece que sólo se ha evaluado

e-ISSN:2172-7910

Doi: 10.25267/Hachetetepe.2021.i23.2205

Universidad de Cádiz 
el estudiantado de una sola sede, por lo que no se pueden discriminar las variables estudiadas entre centros

El modelo de enseñanza más utilizado ha sido la asociación de gamificación con flipped classroom, aunque dos de los estudios corresponden al mismo grupo de investigación, liderado por Gómez-Carrasco $(2019,2020)$. En este modelo, en general, la gamificación se considera como una actividad que tiene poco que ver con las tensiones y deberes de la vida diaria; al contrario, ofrece un escape de ellos junto con la metodología del "aula invertida". Esta percepción se basa en el paradigma de la oposición entre el "mundo del juego" y el "mundo del trabajo" (en términos de fenomenología social). En este doble contexto (gamificación y flipped classroom), la gamificación elimina esta oposición, exponiendo el hecho de que la misma actividad puede percibirse como juego o como trabajo, dependiendo de la forma en que se presente (como una actividad voluntaria u obligatoria) ya que el problema no está en la actividad en sí, sino en nuestra actitud hacia ella. Estos resultados confirman lo indicado por Lledó et al., (2016) según los cuales, el flipped learning favorecía los conocimientos de los estudiantes y su grado de satisfacción. Por tanto, se puede afirmar que las actividades realizadas fuera del aula permiten asimilar los contenidos de las clases y mejoran la implicación del alumnado en el aprendizaje, Además, también se mejora el aprendizaje gracias las rúbricas, a las autoevaluaciones y a los ejercicios de reflexión, conduciendo a una mejora de las calificaciones.

Por otro lado, la gamificación reúne cualidades del juego transcendentes ya que, al menos, en 7 de los trabajos seleccionados, la gamificación mejora tanto el rendimiento académico, como el aprendizaje significativo, la adquisición de competencias y el nivel de conocimientos, términos diferentes empleados en los artículos pero que, sin duda, se encuentran juntos en la mejora del aprendizaje.

A pesar de la amplia implantación en las universidades españolas, Moodle sólo ha sido estudiada por Santos-Villalba et al., (2020) los cuales concluyeron que mejoraba la motivación y, levemente, algunos criterios de sostenibilidad. Corrobora los datos de De Lucas-Santos (2017) que indicaba que el uso de Moodle suponía una herramienta de apoyo docente para la obtención de evaluaciones válidas y rápidas, para efectuar un aprendizaje motivador y continuo en el estudiantado y desarrollar un ámbito de colaboración y cooperación, especialmente con el empleo del foro apoyado con rúbricas.

Una de las objeciones a las que se enfrenta la gamificación es el campo de conocimiento en el que se aplica. ¿Son ciertos campos o temas más accesibles para juegos y simulaciones digitales que no digitales? La gamificación se empleó con estudiantes de Educación Primaria en 6 de los estudios seleccionados, pero su utilidad se ha evaluado en distintos campos de conocimiento que abarcan las ciencias sociales, tecnológicas y sanitarias, que justifica el uso transversal de la gamificación en diferentes áreas de conocimiento.

Las técnicas más frecuentes de evaluación de los efectos de la gamificación han sido las encuestas y cuestionarios $(n=11)$, posiblemente porque la gamificación permite crear una narrativa atractiva que avanza conforme progresa la encuesta, y es suficientemente atractiva como para evitar el abandono. Además, la gamificación permite incluir elementos de feedback y elementos de refuerzo positivo para premiar a los usuarios por responder a las preguntas o indicarles su progreso e, incluso, la gamificación permite que los usuarios suban de nivel con cada encuesta respondida, o ganen 
recompensas virtuales por responder a las encuestas o los formularios a tiempo, premiando así la puntualidad y la diligencia (Seaborn y Fels, 2015).

La amplia gama de plataformas de gamificación empleada en los estudios seleccionados muestra la diversidad de usos de la gamificación y la multitud de herramientas que pueden ser útiles para promover la motivación y las mejoras significativas del aprendizaje. Las limitaciones de los juegos digitales, como los gastos o el conocimiento técnico, pueden disminuir a medida que los motores de diseño de juegos continúan desarrollándose exponencialmente (Wiggins, 2016). En la literatura, las herramientas que tienen más consenso son Kahoot!, Socrative y Quizizz, aunque esta última no se ha utilizado en ninguno de los estudios seleccionados y puede ser la herramienta de gamificación que ofrece mayores beneficios al profesor siendo un software gratuito y fácilmente accesible tanto para los docentes como para los alumnos (Heredia-Sánchez et al., 2020). Respecto a la plataforma Kahoot! utilizada en 3 de los estudios seleccionados, facilita la gamificación alineándose con la teoría de Thomas Malone de instrucciones intrínsecamente motivadoras (Malone, 1980). Esta teoría indica tres categorías que hacen que el aprendizaje sea divertido: 1) desafío (metas con resultados inciertos), 2) fantasía (captación a través de fantasías intrínsecas o extrínsecas) y 3 ) curiosidad (curiosidad sensitiva a través de gráficos y audios, y curiosidad cognitiva). Como la teoría se contextualiza dentro del entorno de aprendizaje del aula, la segunda categoría, la fantasía, se transforma en un programa de juegos con el profesor y los estudiantes desempeñando el papel de presentadores y competidores, respectivamente. La categoría inicial, desafío, implica que los estudiantes sean desafiados a responder preguntas y competir contra otros jugadores. La categoría final, curiosidad, se muestra a través de gráficos y audio y hace que los estudiantes resuelvan acertijos cognitivos (Tan Ai Lin et al., 2018).

No obstante, se deben tener en cuenta determinadas limitaciones que supone la implementación de las metodologías activas por medio de las TIC. Lo más trascendental en este sentido es que el profesorado debe motivar al alumnado, debido a que ambos requieren de una motivación alta. La perspectiva que el alumnado ha manifestado es que, aunque la carga de trabajo no ha sido mayor en lo que respecta a las metodologías tradicionales, el trabajo sí que ha sido considerado más complejo, por lo que el estudiantado ha necesitado un tiempo de dedicación mayor en tareas determinadas. Por otra parte, conforme las actividades se van complicando, la desmotivación estudiantil aumenta y esto debe ser tenido en cuenta y controlado por parte del profesorado. Desde el punto de vista de los docentes, la carga de trabajo se ve elevada considerablemente, sobre todo en las primeras aplicaciones, debido a que el seguimiento y orientación inicial suponen la clave del éxito. Igualmente, ofrecer un feedback en forma y tiempos adecuados supone también un mayor tiempo de dedicación.

En definitiva, a pesar de las limitaciones citadas que se encuentran en la implementación de las metodologías activas, que los docentes aprovechen adecuadamente las plataformas e-learning como herramienta de apoyo al proceso de EA, indica sólo las ventajas que supone la modernización de la enseñanza universitaria por medio de las modificaciones de roles, de la formación continua del profesorado y adaptada a las exigencias de la sociedad y de los futuros docentes que están formándose.

Palazón-Pérez (2011) indicaban que las metodologías de autoaprendizaje activo en las universidades eran limitadas. 
Por último, la gamificación y el aprendizaje basado en juegos no solo son áreas que merecen una mayor investigación, sino que pueden revelar una forma de que la educación superior combata la disminución de la matrícula mediante el uso innovador de juegos interactivos y simulaciones dentro del aula y la experiencia educativa superior en general.

\section{CONCLUSIONES}

Las gamificación sola o asociada a otras metodologías como flipped classroom está siendo objeto de numerosas investigaciones en el marco universitario español, aunque su implantación aún es menor que en otros países culturalmente relacionados.

La docencia basada en juegos no se limita a grados de Educación, sino que se aplica tanto en grados sociales, como técnicos y sanitarios.

Los estudios seleccionados muestran que la gamificación contribuye a mejorar la motivación del alumnado y puede tener efectos positivos, entre otros, con el rendimiento académico y una amplia gama de competencias universitarias.

No existe consenso en cuanto a las fuentes de gamificación. Es necesario seguir estudiando los efectos de la gamificación en el entorno universitario, especialmente en relación a la amplia gama de plataformas utilizadas.

El profesorado universitario debe seguir formándose en las herramientas que sustentan la gamificación, como las TIC y las plataformas docentes tipo Moodle.

CONTRIBUCIÓN DE LOS AUTORES: Santiago Alonso-García (conceptualizacón), José Antonio Martínez-Domingo (Tratamiento de datos), Blanca Berral-Ortiz (Análisis formal) y Juan Carlos De la Cruz-Campos (Adquisición de fondos, investigación).

FINANCIACIÓN: Esta investigación no recibió ninguna financiación externa.

\section{REFERENCIAS BIBLIOGRÁFICAS}

Ardila-Muñoz, J.Y. (2019). Supuestos teóricos para la gamificación de la educación superior. Magis, Revista Internacional de Investigación en Educación, 12(24), 7184. https://doi.org/10.11144/Javeriana.m12-24.stge

Area-Moreira, M., Bethencourt-Aguilar, A., Martin-Gomez, S., y Nicolas-Santos, S. (2021). Analysis of Higher Education Policies in Spain for Covid-19: Adapted Presence. RED-Revista De Educacion a Distancia, 21(65), 1-19. https://doi.org/10.6018/red.450461

Attali, Y., y Arieli-Attali, A. (2015). Gamification in assessment: do points affect test performance? Computers and Education, 83(1), 57-63. https://doi.org/10.1016/j.compedu.2014.12.012

Berenguer, C. (2016). Acerca de la utilidad del aula invertida o flipped classroom. En M.T. Tortosa, S. Grau, y J.D. Álvarez. (Ed.), XIV Jornadas de redes de investigación en docencia universitaria. Investigación, innovación y enseñanza universitaria: enfoques pluridisciplinares (pp. 1466- 1480). Universitat d'Alacant.

Borras-Gene, O., Martínez-Núñez, M., y Fidalgo-Blanco, Á. (2016). New challenges for the motivation and learning in engineering education using gamification in MOOC. International Journal of Engineering Education, 32(1B), 501-512. 
Bravo-Lucas, E., Costillo-Borrego, E., Bravo-Galán, J.L., y Borrachero-Cortés, A.B. (2020). Emociones de los futuros maestros de educación infantil en las distintas áreas del currículo. Profesorado. Revista de Currículum y Formación de Profesorado, 24(1), 96-114. https://doi.org/10.30827/profesorado.v24i1.8846

Bullón, J.J., Encinas, A.H., Sánchez, M.J.S., y Martínez, V.G. (2018). Analysis of student feedback when using gamification tools in math subjects. En IEEE Global Engineering Education Conference (EDUCON) (pp. 1818-1823). IEEE.

Campillo-Ferrer, J.M., Miralles-Martínez, P., y Sánchez-Ibáñez, R. (2020). Gamification in higher education: Impact on student motivation and the acquisition of social and civic key competencies. Sustainability, 12(12), 1-13. https://doi.org/10.3390/su12124822

Cohen, L., y Manion, L. (2001). Research Methods in Education 5th Edition. Rotledge Falmer.

Cózar-Gutiérrez, R., y Sáez-López, J. M. (2016). Game-based learning and gamification in initial teacher training in the social sciences: an experiment with MinecraftEdu. International Journal of Educational Technology in Higher Education, 13(2), 111. https://doi.org/10.1186/s41239-016-0003-4

Csikszentmihalyi, M. (2005). Fluir (flow): una psicología de la felicidad. Kairós.

Cuevas-Martínez, J. C., Yuste-Delgado, A.J., Pérez-Lorenzo, J.y M., y Triviño-Cabrera, A. (2019). Jump to the next level: A four-year gamification experiment in information technology engineering. IEEE Access, 7, 118125-118134. https://doi.org/10.1109/ACCESS.2019.2932803

De Lucas-Santos, S. (2017). El uso de las TIC para el desarrollo de competencias con metodologías activas en Estadística Descriptiva del grado de ADE. REDU. Revista de docencia universitaria, 15(2), 245-256. https://doi.org/10.4995/redu.2017.7405

Deci, E.L. y Ryan, R.M. (1985). Intrinsic motivation and self-determination in human behavior. Springer.

Duran, D. (2010). Cooperative Interactions in Peer Tutoring: Patterns and Sequences in Paired Writing. Middle Grades Research Journal, 5(1), 47-60.

Fernández-Antolín, M.M., del Río, J.M., y González-Lezcano, R.A. (2020). The use of gamification in higher technical education: perception of university students on innovative teaching materials. International Journal of Technology and Design Education, 1-20. https://doi.org/10.1007/s10798-020-09583-0

Fernández-Solo de Zaldívar, I. (2015). Juego serio: gamificación y aprendizaje. Comunicación y Pedagogía. Nuevas tecnologías y recursos didácticos, (281-282), 43-48.

Ferriz-Valero, A., Østerlie, O., García Martínez, S., y García-Jaén, M. (2020). Gamification in physical education: Evaluation of impact on motivation and academic performance within higher education. International Journal of Environmental Research and Public Health, 17(12), 1-16. https://doi.org/10.3390/ijerph17124465

Galbis-Córdoba, A., Martí-Parreño, J., y Currás-Pérez, R. (2017). Education Students' Attitude towards the Use of Gamification for Competencies Development. Journal of e-Learning and Knowledge Society, 13(1), 129-146. https://doi.org/10.20368/1971-8829/158 
García-Holgado, A., García-Peñalvo, F.J., de la Higuera, C., Teixeira, A., Ehlers, U.D., Bruton, J., Nascimbeni, F., Padilla, N., y Burgos, D. (2020). Promoting Open Education Through Gamification in Higher Education: the OpenGame project. En F.J. García-Peñalvo y A. García-Holgado (Eds.) Eighth International Conference on Technological Ecosystems for Enhancing Multiculturality (pp.399-404). Association for Computing. https://doi.org/10.1145/3434780.3436688

Gay, A.S., y Burbridge, L. (2016). "Bring your own device" for formative assessment. The Mathematics Teacher, 110(4), 310-313. https://doi.org/10.5951/mathteacher.110.4.0310

Gómez-Carrasco, C. J., Monteagudo-Fernández, J., Moreno-Vera, J. R., y Sáinz-Gómez, M. (2020). Evaluation of a gamification and flipped-classroom program used in teacher training: Perception of learning and outcome. PloS one, 15(7). https://doi.org/10.1371/journal.pone.0236083

Gómez-Carrasco, C.J., Monteagudo-Fernández, J., Moreno-Vera, J.R., y Sáinz-Gómez, M. (2019). Effects of a gamification and flipped-classroom program for teachers in training on motivation and learning perception. Education Sciences, 9(4),1-15. https://doi.org/10.3390/educsci9040299

Gómez, I. M. y Ruiz, M. (2017). Análisis de metodologías activas con ABP, Transmedia y Gamificación para implementar las competencias en Ciencias Sociales y en Literatura. En R. Roig-Vila (Ed.), Investigación en docencia universitaria. Diseñando el futuro a partir de la innovación educativa (pp.245-254). Octaedro.

Haruna, H., Hu, X., Chu, S.K.W., Mellecker, R.R., Gabriel, G., y Ndekao, P.S (2018). Improving Sexual Health Education Programs for Adolescent Students through Game-Based Learning and Gamification. International Journal of Environmental Research and Public Health, 15(9), 1-26. https://doi.org/10.3390/ijerph15092027

Heredia-Sánchez, B.D.C., Pérez-Cruz, D., Cocón-Juárez, J.F., y Zavaleta-Carrillo, P. (2020). La Gamificación como Herramienta Tecnológica para el Aprendizaje en la Educación Superior. Revista Tecnológica-Educativa Docentes 2.0, 9(2), 49-58.

Hernández-Padrón, I.M. (2018). El Ministerio de Robin Hood: una experiencia de gamificación. Números: Revista de didáctica de las matemáticas, 98, 153-162.

Johnson, R. y Johnson, D. (2000). Active Learning: Cooperation in the College Classroom. The Annual Report of Educational Psychology in Japan, 47, 29-30. https://doi.org/10.5926/arepj1962.47.0_29

Kapp, K.M. (2012). The gamification of learning and instruction: game-based methods and strategies for training and education. Pfeiffer.

Krippendorff, K. (2018). Content analysis: An introduction to its methodology. Sage publications.

Lee, J. y Hammer, J. (2011). Gamification in education: What, how, why bother? Academic Exchange Quarterly, 15(2), 1-5.

Lledó, A., Lorenzo, G., Arráez, G., Lorenzo, A., Gonzálvez, C., Gómez, M., Leal, M., Sanmartín, R. y Vicent, M. (2016) Una propuesta de modelo para introducir metodologías activas en educación superior. En J.M. Antolí, A. Lledó, y N. Pellín. (Eds.), Memorias del Programa de Redes-I CE de calidad, innovación e investigación en docencia universitaria. Convocatoria 2016-17 (pp. 2702-2712). Instituto de Ciencias de la Educación (ICE) de la Universidad de Alicante. 
Malone, T.W. (1980). What makes things fun to learn? Heuristics for designing instructional computer games. En The 3rd ACM SIGSMALL Symposium and the 1st SIGPC Symposium on Small Systems (pp. 162-169). ACM Press.

Maquilón, J., Sánchez, M. y Cuesta, J. (2016). Enseñar y aprender en las aulas de Educación El impacto de las metodologías activas en los resultados académicos 114 Primaria. Revista Electrónica de Investigación Educativa, 18(2), 144-155.

Marín-Díaz, V. (2015). La gamificación educativa. Una alternativa para la enseñanza creativa. Digital Education Review, (27), 1-4. https://doi.org/10.1344/der.2015.27.\%25p

Méndez-Giménez, A., Fernández-Río, J., Rolim-Marques, R.J. y Calderón, A. (2016). Percepciones de estudiantes de máster en Educación Física acerca de los materiales autoconstruidos. Una mirada desde la teoría construccionista de Papert. Educación XX1, 19(1), 179-200. https://doi.org/10.5944/educXX1.15583

Moher, D., Liberati, A., Tetzlaff, J., Altman, D.G., The PRISMA Group (2009). Preferred Reporting Items for Systematic Reviews and Meta-Analyses: The PRISMA Statement. PLoS Med, 6(7), 1.-6. https://doi.org/10.1371/journal.pmed.1000097

Murillo-Zamorano, L.R., López-Sánchez, J.A., y Godoy-Caballero, A.L. (2017). Gamification in higher education. A creative and technological proposal. En Innovative and Creative Education and Technology (pp.158-161). ICETIC

Ortega, D. y Gómez, I.Ma . (2017). Las WebQuests y los MOOC's en la enseñanza de las Ciencias Sociales y la formación del profesorado de Educación Primaria. Revista Interuniversitaria de Formación del Profesorado, 20(1), 57-73. https://doi.org/10.6018/reifop/20.2.258551

Palazón-Pérez, A., Gómez-Gallego, M., Gómez-Gallego, J.C., Pérez-Cárceles, M.C. y Gómez-García, J. (2011). Relación entre la aplicación de metodologías docentes activas y el aprendizaje del estudiante universitario. Bordón. Revista de pedagogía, 63(2), 27-40. https://bit.ly/3iuwBNr

Rodríguez-Gómez, G., Ibarra-Saiz, M.S. y Cubero-Ibáñez, J. (2018). Competencias básicas relacionadas con la evaluación. Un estudio sobre la percepción de los estudiantes universitarios. Educación XX1, 21(1), 181-208. https://doi.org/10.5944/educxx1.20184

Rovira-Collado, J., Llorens, R.F. y Fernández, S. (2016). Una propuesta transmedia para la educación literaria: el ministerio del tiempo. En M.T. Tortosa, S. Grau Company, y J.D. Álvarez Teruel, (Coords.) XIV Jornadas de Redes de Investigación en Docencia Universitaria. Investigación, innovación y enseñanza universitaria: enfoques pluridisciplinares. (pp. 569-584) Universitat d'Alacant, ICE.

Ruiz-Alba, J., Soares, A., Rodríguez-Molina, M., y Banoun, A. (2019). Gamification and entrepreneurial intentions. Journal of Small Business and Enterprise Development, 26(5), 661-683. https://doi.org/10.1108/JSBED-09-2018-0266

Ryan, R.M. y Deci, E.L. (2000). Intrinsic and Extrinsic Motivations: Classic Definitions and New Directions. Contemporary Educational Psychology, 25(1), 54-67. https://doi.org/10.1006/ceps.1999.1020

Sánchez-Mena, A., y Martí-Parreño, J. (2016). Gamification in higher education: teachers' drivers and barriers. En Proceedings of the International Conference the Future of Education (pp. 180-184). 
Santos-Villalba, M.J., Leiva Olivencia, J.J., Navas-Parejo, M.R., y Benítez-Márquez, M. D. (2020). Higher Education Students' Assessments towards Gamification and Sustainability: A Case Study. Sustainability, 12(20), 1-20. https://doi.org/10.3390/su12208513

Seaborn, K., y Fels, D.I. (2015). Gamification in theory and action: A survey. International Journal of human-computer studies, 74, 14-31. https://doi.org/10.1016/j.ijhcs.2014.09.006

Tan Ai Lin, D., Ganapathy, M. y Kaur, M. (2018). Kahoot! It: Gamification in Higher Education. Pertanika Journal of Social Sciences \& Humanities, 26(1), 565-582.

Teixes, F. (2014). Gamificación: fundamentos y aplicaciones. Universitat Oberta de Catalunya.

Torres-Toukoumidis, A., Valle-Razo, A.L., y De Santis, A. (2020). Gamification in Higher Education Instructors from Ecuador, Spain and Mexico. En 2020 IEEE ANDESCON (pp. 1-4). IEEE.

Werbach, K. y Hunter, D. (2014). Gamificación revoluciona tu negocio con las técnicas de los juegos. Pearson Educación.

Wiggins, B. E. (2016). An overview and study on the use of games, simulations, and gamification in higher education. International Journal of Game-Based Learning (IJGBL), 6(1), 18-29. https://doi.org/10.4018/IJGBL.2016010102

Zarzycka-Piskorz, E. (2016). Kahoot it or not? Can games be motivating in learning grammar? Teaching English with Technology, 16(3), 17-36. 